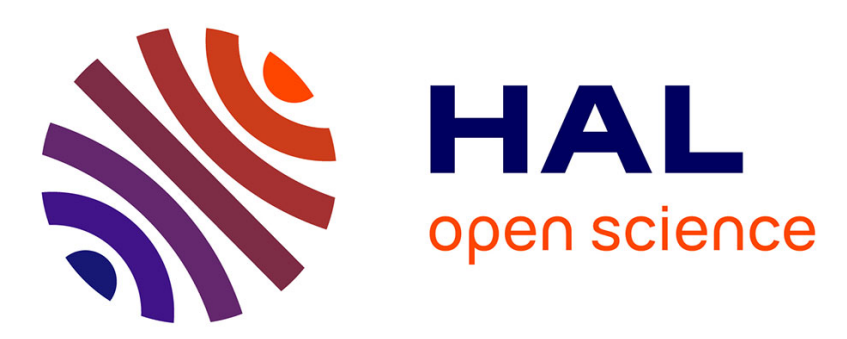

\title{
Astyanax et les orphelins de guerre athéniens. Critique de l'idéologie de la cité dans les Troyennes d'Euripide
}

Pascale Brillet-Dubois

\section{To cite this version:}

Pascale Brillet-Dubois. Astyanax et les orphelins de guerre athéniens. Critique de l'idéologie de la cité dans les Troyennes d'Euripide. Revue des Etudes Grecques, 2010, 123 (1), pp.29-50. hal-01464582

\section{HAL Id: hal-01464582 \\ https://hal.science/hal-01464582}

Submitted on 10 Feb 2017

HAL is a multi-disciplinary open access archive for the deposit and dissemination of scientific research documents, whether they are published or not. The documents may come from teaching and research institutions in France or abroad, or from public or private research centers.
L'archive ouverte pluridisciplinaire HAL, est destinée au dépôt et à la diffusion de documents scientifiques de niveau recherche, publiés ou non, émanant des établissements d'enseignement et de recherche français ou étrangers, des laboratoires publics ou privés. 
Pascale BRILLET-DUBOIS

\author{
« ASTYANAX ET LES ORPHELINS DE \\ GUERRE ATHÉNIENS. \\ CRITIQUE DE L'IDÉOLOGIE DE LA CITÉ \\ DANS LES TROYENNES D'EURIPIDE $\gg^{1}$
}

A la mémoire de Pierre Vidal-Naquet

Résumé. - « Astyanax et les orphelins de guerre athéniens. Critique de l'idéologie de la cité dans les Troyennes d'Euripide »

La question de la portée polémique des Troyennes d'Euripide a été abordée récemment soit du point de vue de l'allusion historique aux événements contemporains (prise de Mélos et préparation de l'expédition de Sicile), soit dans la perspective intellectuelle des débats sophistiques, soit en mettant l'accent sur le pouvoir subversif de la lamentation féminine. On s'intéresse ici, pour infirmer ou compléter certaines de ces hypothèses antérieures, à l'usage que fait la pièce de discours et de rites officiels qui expriment ou matérialisent l'idéologie guerrière athénienne, notamment dans l'enceinte même du théâtre au cours des Grandes Dionysies, dans les jours précédant le concours tragique. La mise en scène et en mots du meurtre d'Astyanax, en particulier, constitue une inversion et une perversion rigoureuses de la cérémonie honorant les orphelins de guerre athéniens. La mort de l'enfant se révèle être non seulement un admirable coup de théâtre, une

\footnotetext{
1 Je remercie tous les collègues et les étudiants qui m'ont aidée en discutant et critiquant les premières versions de cet article, présentées dans plusieurs Universités américaines (UC Davis, Stanford University, UC Santa Cruz, University of Florida), ainsi que Christine Mauduit, Nadine Le Meur-Weissman et Patrick Lee Miller pour leur relecture attentive. Ce travail a été rendu possible grâce au soutien financier et intellectuel du Harvard Center for Hellenic Studies, Washington DC, dont je remercie vivement le directeur, Greg Nagy, le personnel et les fellows.
} 
scène extrêmement pathétique suscitant une pitié universelle, mais aussi l'instrument d'une charge politique ancrée dans un contexte historique bien déterminé, ayant pour cible l'idéologie guerrière d'Athènes dont elle révèle les failles et les dangers.

\section{Abstract. - « Astyanax and the Athenian War Orphans. Challenging the City's Ideology in Euripides' Trojan Women »}

When attempting to assess the critical strenghth of Euripides' Trojan Women, recent scholarship has focused mainly on three features: 1) allusion to contemporary events (the war against Melos and the Sicilian expedition), 2) polemical speeches and arguments, 3) subversive power of female lamentation. As a contribution to that discussion, the present paper dwells on the way the play integrates official Athenian discourses and ceremonies that express or enact the city's war ideology, especially the ones that were heard and seen in the theatre during the Great Dionysia before the tragic competition started. The murder of Astyanax, in particular, appears to be a precise inversion, in words and action, of the ceremony honoring Athens' war orphans. Therefore the child's death is not only the much praised, extremely pathetic coup de théâtre that spectators universally meet with pity; it also constitutes a political statement against Athenian war ideology by revealing its flaws and potential destructiveness.

Parmi les tragédies grecques qui nous sont parvenues, Les Troyennes semblent réfléchir de façon particulièrement efficace les angoisses et les souffrances de notre époque. La description pathétique que fait Euripide de la manière dont la guerre affecte une communauté, et en particulier les plus vulnérables de ses membres, les femmes et les enfants, éveille spontanément la compassion et offre un support à bien des dénonciations ou des protestations. La pièce a été régulièrement traduite et mise en scène au siècle dernier pour ses analogies frappantes avec la plupart des grands conflits qui ont agité le monde et influencé le travail des philologues et des metteurs en scène occidentaux : la guerre des Boers, la Seconde Guerre Mondiale, les conflits d'Algérie ou du Vietnam, la lutte contre la dictature des colonels en Grèce, la guerre en $\operatorname{Irak}^{2}$... Mais si universels que puissent paraître le sujet et les personnages de la pièce, si fructueuses, si créatives que soient ses nombreuses adaptations, il me semble qu'il reste encore beaucoup à dire et à dévoiler sur le contexte de création de cette tragédie, dans lequel elle prend toute sa signification et toute sa force.

Dans son article intitulé «The Great Dionysia and Civic Ideology », publié dans une collection d'essais désormais classique consacrée au théâtre athénien ${ }^{3}$, Simon Goldhill a examiné la relation entre

\footnotetext{
2 Voir Goff, B., Euripides. Trojan Women, Londres, 2009, p. 78-135.

3 Winkler, J., Zeitlin, F., Nothing to Do with Dionysos? Athenian Drama in its Social Context, Princeton, 1990, p. 97-129.
} 
les concours dramatiques et la série de cérémonies qui les précédaient durant les Grandes Dionysies. Il entendait montrer qu'au cours de cette fête, l'idéologie de la cité, son identité ( « the city's sense of self »), était d'abord affirmée publiquement, avant d'être mise en cause dans la tragédie et la comédie : « les libations des dix stratèges, l'exposition du tribut, la proclamation de la liste des bienfaiteurs de la cité, la parade, en tenue militaire complète, de garçons éduqués aux frais de l'Etat et parvenus à l'âge d'homme [les orphelins de guerre] ${ }^{4}$, tout cela soulignait le pouvoir de la polis et les obligations des individus envers la polis $»^{5}$. S. Goldhill poursuivait en analysant la manière dont cette juxtaposition devait influencer l'interprétation de l'Ajax et du Philoctète de Sophocle et mettait en évidence le fait que certaines des valeurs, certains des devoirs imposés par la cité démocratique et mis en cause dans les deux pièces étaient précisément ceux qui avaient été si vigoureusement exaltés dans le théâtre-même juste avant les représentations.

Je voudrais montrer que, dans les Troyennes, Euripide se livre à une critique similaire de certains discours et cérémonies fondamentaux de l'idéologie guerrière athénienne et, en particulier, qu'il fait un usage dramatique tout à fait semblable, sinon plus radical, du contexte des Dionysies dans lequel est représentée la tragédie : le meurtre d'Astyanax est en effet présenté comme une inversion systématique de la cérémonie des orphelins de guerre athéniens et il se peut que les dépouilles troyennes fassent allusion au tribut des alliés. L'examen de ce lien entre la tragédie et les rites civiques qui l'ont précédée m'amènera à reconsidérer la force critique et le pouvoir de provocation de la pièce.

L'une des tendances récentes des études sur cette tragédie est de voir dans la lamentation rituelle - omniprésente dans les Troyennes - et dans le lyrisme féminin son aspect le plus subversif ${ }^{6}$. Il y a sans doute là une réaction à des travaux comme celui de $\mathrm{N}$. Croally ${ }^{7}$, centrés principale-

${ }^{4}$ Sur ces cérémonies, voir Csapo, E., Slater, W.J., The Context of Ancient Drama, Ann Arbor, 1995, p. 117-119. A.W. Pickard-Cambridge (The Dramatic Festivals of Athens, Londres, $1968^{2}$, p. 67) et J.-Ch.Moretti (Théâtre et société dans la Grèce antique, Paris, 2001, p. 83-84) les mentionnent brièvement.

5 «The libations of the ten generals, the display of tribute, the announcement of the city's benefactors, the parade of state-educated boys [the war orphans], now men, in full military uniform, all stressed the power of the polis, the duties of an individual to the polis » (Goldhill, S., « The Great Dionysia and Civic Ideology », p. 114).

${ }^{6}$ Voir Alexiou, M., The Ritual Lament in Greek Tradition, Lanham, MD, 2002², et à sa suite Loraux, N., La voix endeuillée : Essai sur la tragédie grecque, Paris, 1999 ; Suter, A., « Lament in Euripides' Trojan Women », Mnemosyne LVI/1, 2003, p. 1-28 ; Dué, C., The Captive Woman's Lament in Greek Tragedy, Austin, 2006.

7 Croally, N., Euripidean Polemic. The Trojan Women and the Function of Tragedy, Cambridge, 1994. 
ment sur les discours et les arguments mis en œuvre de manière polémique par Euripide. Il me semble qu'il faut tenter de réconcilier les aspects politiques et intellectuels de la pièce avec l'insistance sur le deuil féminin, tout en faisant justice à l'art dramatique d'Euripide, à son sens du spectacle et de la scène. Ce que je voudrais montrer dans le cas d'Astyanax, c'est que l'idéologie guerrière athénienne est incorporée à la tragédie, non pour y être réfutée par des arguments, mais pour y être confrontée à l'expérience des victimes et fournir le cadre et la matière d'un spectacle profondément dérangeant.

Les Troyennes sont la troisième pièce d'une trilogie consacrée par Euripide à la guerre de Troie, les deux premières pièces étant Alexandros et Palamède dont nous n'avons conservé que des fragments ${ }^{8}$. R. Scodel, dans l'ouvrage le plus complet sur les trois pièces ${ }^{9}$, conclut de façon persuasive en faveur d'une trilogie liée. L'Alexandros et les Troyennes ont en commun plusieurs personnages : Hécube et Cassandre apparaissent dans les deux pièces, tandis que le souvenir d'Hector, de Priam et de Pâris, présents dans la première, revient constamment dans la seconde. Ils sont aussi liés tant par les thèmes développés noblesse et esclavage, Grecs et barbares, victoire et défaite,... - que par l'intrigue - le rôle tragique de Pâris dans la chute de Troie est prédit dans l'Alexandros, tandis que la mort prématurée d'Astyanax, sur laquelle se clôt Les Troyennes, s'oppose et répond à la survie de Pâris enfant, objet de la première pièce. Les rapports avec le Palamède, très fragmentaire, sont plus délicats à établir, mais nous sommes du moins certains que son intrigue se déroule, comme les deux autres pièces, à Troie (en l'occurrence, dans le camp des Achéens), dans le temps qui sépare les événements de la première pièce de ceux de la troisième, et qu'Ulysse, dont l'influence néfaste se fait sentir sur les Troyennes, en est l'un des personnages principaux.

Quand la trilogie fut représentée en 415 av. J-C, cela faisait des années qu'Athènes était en guerre, soit contre Sparte, soit pour le maintien de son empire, et la cité se préparait à la grande expédition de Sicile qui allait la conduire au désastre. Les citoyens assemblés ce printemps-là au théâtre pour les Grandes Dionysies étaient physiquement et moralement meurtris par la guerre, et plus d'un spectateur avait probablement perdu un père, un frère ou un fils au combat. Mais les Athéniens avaient aussi commis des massacres dans plusieurs cités du monde grec, le plus récent étant celui de Mélos dans les derniers mois

8 Voir Jouan, F., Van Looy, H., éd. Euripide. Fragments, vol. 1 et 2, CUF, Paris, 1998 et 2000 .

9 Scodel, R., The Trojan Trilogy of Euripides, Göttingen, 1980. 
de l'année 416, où ils avaient éliminé la population masculine et asservi femmes et enfants : la cité refusait de rejoindre la ligue de Délos et souhaitait rester neutre. Les commentateurs des Troyennes débattent toujours pour savoir si Euripide fait une référence directe à ces événements ${ }^{10}$; mon but n'est pas de résoudre cette question, bien que, comme nous le verrons, certaines des idées développées plus loin lui soient liées. Il me semble que, quelle qu'ait été l'intention d'Euripide au moment où il composait la tragédie, il est plus que probable qu'au moins une partie des spectateurs aient fait le lien entre ce qui se passait sur la scène et ce qu'ils vivaient par ailleurs. Au sein du public, certains ont dû voir dans le sort des mythiques Troyens une réécriture, voire une sublimation de celui des Méliens. Pour autant, je ne crois pas qu'on puisse se contenter d'assimiler les Troyens aux Méliens et les Grecs aux Athéniens. Une lecture allégorique de ce type pèche par excès de simplicité. En effet, du fait de ses caractéristiques mythiques et de l'influence de l'Iliade, Troie pouvait incarner à la fois une cité barbare lointaine, située au-delà des mers, objet de haine et de convoitise pour des Grecs qui l'avaient finalement vaincue et pillée, et une cité combattant vaillamment pour la défense de ses murs, de ses femmes, de ses enfants. Les Athéniens pouvaient ainsi à la fois, ou tour à tour, la considérer, de l'œil des conquérants, comme une proie et une ennemie, ou s'identifier avec elle en tant que citoyens-soldats passionnément attachés à la défense de leur propre communauté ${ }^{11}$.

La structure des Troyennes est simple. Quand la pièce débute, Troie a été vaincue par les Grecs, les hommes et les guerriers troyens sont tous morts et les femmes attendent d'être attribuées à leur nouveau maître. Les bateaux des vainqueurs sont sur le point de prendre la mer et de les emporter vers la servitude et l'exil. Dans le prologue, le dieu Poséidon apparaît pour prendre congé de la cité. Il quitte le sanctuaire, désormais désert, où les Troyens l'honoraient et lui faisaient des offrandes. Athéna, victorieuse de son oncle dans le conflit divin qui faisait rage autour de Troie, le rejoint en scène pour lui demander une faveur : elle veut maintenant détruire les Grecs, car bien qu'elle les ait conduits à la victoire, ils l'ont offensée, et elle souhaite déclencher contre leurs

10 Voir les synthèses de cette controverse par P. Burian (Burian, P., Shapiro, A., Euripides. Trojan Women, Oxford, 2009, p. 4-6) et B. Goff (Goff, Trojan Women, p. 27-35). L'interprétation historiciste de la pièce et de la trilogie est défendue notamment par E. Delebecque (Euripide et la guerre du Péloponnèse, Paris, 1951, p. 245-262) et R. Goossens (Euripide et Athènes, Bruxelles, 1962, p. 507-538), et critiquée radicalement par G. Zuntz («Contemporary politics in Euripides », in Opuscula Selecta. Classica, Hellenistica, Christiana, Manchester, 1972, p. 54-61). Conclusion plus modérée chez N. Croally, p. 231-234.

11 Voir Dué C., The Captive Woman's Lament, p. 91-116. 
navires une tempête fatale. Poséidon accepte de l'aider, mais le désastre grec n'aura pas lieu dans le temps de la pièce. Au lieu de cela, ce sont la distribution et le départ des princesses troyennes - les plus précieuses des captives, celles qui ont été choisies par leur maître (exairetoi) et non attribuées par le sort - qui constituent l'action principale. Cassandre est la première : Agamemnon veut l'épouser au mépris de son statut de prêtresse d'Apollon et de vierge consacrée. Puis c'est le tour d'Andromaque, la veuve d'Hector, meilleur des Troyens : elle revient, avec son fils Astyanax, à Néoptolème, meilleur des Achéens, mais aussi fils d'Achille, le meurtrier d'Hector. Hélène partage le sort des captives, mais c'est Ménélas en personne qui vient la chercher - et non le héraut Talthybios comme les autres. Bien qu'Hécube le convainque de sa culpabilité, il ne peut se résoudre à la châtier immédiatement. Enfin, ce sont Hécube, destinée au palais d'Ulysse, et les captives du chœur que les gardes viennent arracher à leur patrie. Un seul événement vient interrompre le cours implacable de cette dispersion des Troyennes : les Grecs ont subitement décidé de tuer Astyanax. Lors du quatrième et dernier épisode, Hécube est autorisée à le pleurer et à préparer son corps pour l'enterrement avant d'être emmenée. A la fin de la tragédie, la communauté de Troie est définitivement anéantie. Le dernier garçon de la famille, qui incarnait le futur de la cité et aurait pu, un jour, refonder Troie, a été tué ; toutes les femmes ont été séparées et sont sur le point d'intégrer des maisons grecques où, sans doute, elles donneront à leur maître des enfants grecs - elles ont dansé leur dernier chœur et seront privées à jamais du réconfort des larmes et des souvenirs partagés ; Troie elle-même est réduite en cendres ; les dieux sont partis, et la plaine de Troie, tout comme la scène, est désertée.

L'entrée d'Andromaque et d'Astyanax, au début du deuxième épisode, est un moment scénique remarquable. Bien des commentateurs l'ont relevé12, mais il me semble qu'il demeure des aspects de ce spectacle qui n'ont pas été totalement explorés. Je voudrais ici suggérer différentes lectures possibles, l'une conjecturale, les autres plus fermement étayées, susceptibles d'approfondir notre compréhension de sa force et de sa signification, et partant, d'éclairer l'ensemble de la pièce.

Lorsque la mère et le fils entrent en scène, les femmes du chœur décrivent le tableau qu'elles ont sous les yeux (568-576). Voici la veuve d'Hector, assise sur un chariot étranger, serrant contre son sein son fils orphelin. Autour d'eux gisent les armes du héros et les

\footnotetext{
12 Pour une analyse éclairante mais limitée, voir Dyson, M., Lee, K.H., « The Funeral of Astyanax in Euripides' Troades », JHS 120, 2000, p. 17-33 ; Halleran, M.R., Stagecraft in Euripides, Londres, 1985, p. 11, 60, 96-97. Sur les scènes d'entrée en char, voir Taplin, O., The Stagecraft of Aeschylus, Oxford, 1977, p. 76-77.
} 
dépouilles des Troyens morts, que Néoptolème entend offrir aux dieux à son retour en Phtiotide. L'espace d'un bref et triste instant, les Troyennes recréent l'unité de la famille d'Hector en associant le fils et la mère au nom du héros : Astyanax est appelé ${ }^{~} E \kappa \tau o \rho \circ \varsigma$ ĩvis et

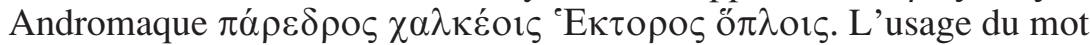
$\pi \alpha ́ \rho \varepsilon \delta \rho o \varsigma$, qui signifie normalement « assis à côté de quelqu'un » ${ }^{13}$, confère à l'armure d'Hector une présence humaine. Il est même plausible que le trio ait été recomposé visuellement, car même si les armes d'Hector ne constituent pas un trophée à proprement parler (elles ne sont pas destinées à être laissées sur le lieu de la défaite troyenne, mais font partie du butin de Néoptolème et doivent être emportées avec lui sur son navire), il est intéressant de noter que, selon l'usage décrit par R. Lonis, les dépouilles servant de trophées étaient disposées de façon anthropomorphe, un peu à la manière d'un épouvantail ${ }^{14}$. Il n'est donc pas impossible d'imaginer que la mise en scène redouble l'effet synecdotique du mot $\pi \alpha ́ \rho \varepsilon \delta \rho o \zeta$ et que les armes d'Hector forment sur le char une figure évoquant un corps humain.

Dans le même temps, tout est fait pour suggérer l'absence d'Hector ${ }^{15}$ - son armure n'est qu'un souvenir creux et vide de l'homme courageux qu'il était - et ses terribles conséquences : la défaite et la mort de ses hommes, l'asservissement de sa femme et de son enfant à un maître qui peut exhiber ses dépouilles en signe de triomphe. Le pitoyable spectacle de la veuve et de l'orphelin, vivants mais terrifiés, au milieu des instruments de mort, souligne à la fois la vanité et le pouvoir destructeur de la violence masculine. Hector, avec toute sa bravoure, a laissé sa femme et son enfant exposés à la brutalité des vainqueurs ${ }^{16}$, et les casques étincelants, les cuirasses et les boucliers sont désormais vidés de la chair des hommes qu'ils protégeaient, comme des peaux de bêtes prises à la chasse ( $\sigma \kappa u ́ \lambda o 1 \zeta . . . \delta o \rho \imath \theta \eta \rho \alpha ́ \tau o 1 \varsigma, 574)$. Ils sont les restes pathétiques de la richesse et de la force des Troyens, le symbole de leur vanité aussi bien que la preuve de leur disparition.

13 L'examen des 23 occurrences du mot aux $\mathrm{VI}^{\mathrm{e}}$ et $\mathrm{V}^{\mathrm{e}}$ siècles av. J.-C. montre que dans 21 cas, il signifie « assis ou siégeant à côté de quelqu'un », qu'il s'agisse d'un dieu ou d'un être humain, au génitif ou au datif. Dans l'un des deux autres cas, il est utilisé à propos de la Pythie, qui siège à côté des aigles d'or de Zeus. Bien que ces aigles puissent être considérés comme des objets, ils ont aussi une valeur métonymique et incarnent la présence de Zeus lui-même, dans un contexte religieux où le mot qualifie généralement deux divinités siégeant côte à côte. Il est donc manifeste que dans le dernier cas, Euripide utilise $\pi \alpha ́ \rho \varepsilon \delta \rho \circ \varsigma$ pour donner à l'armure d'Hector et aux dépouilles troyennes une présence corporelle, humaine.

14 Lonis, R., Guerre et religion en Grèce à l'époque classique : recherches sur les rites, les dieux, l'idéologie de la victoire, Paris, 1979, p. 129.

15 Sur l'absence d'Hector et sa fonction dans la pièce, voir Poole, A., « Total Disaster : Euripides' The Trojan Women », Arion 3/3, 1976, p. 257-287.

16 Talthybios ne se prive pas de le rappeler à Andromaque v. 729 et suivants. 
De même, l'entrée d'Andromaque et d'Astyanax sur le chariot chargé de dépouilles évoque leur chute, le renversement de fortune qui les a fait basculer d'un statut royal et libre à la captivité. Euripide joue notamment sur le contraste avec d'autres moments de théâtre, d'autres tragédies - au premier rang desquelles, sans doute, l'Agamemnon - dans lesquelles des personnages royaux au sommet de leur pouvoir entraient en scène sur des chars ${ }^{17}$. Ici, la mère et le fils ne sont pas mis en valeur par une place prééminente sur le véhicule, mais sont représentés visuellement comme partie du chargement, comme des éléments du butin, avant même qu'Andromaque n'exprime cette idée en mots (614). Rien ne les distingue à première vue des objets inanimés avec lesquels ils sont emportés, avant que le chœur ne les nomme respectueusement et que la princesse troyenne n'entame sa lamentation, énonçant dans le chant sa souffrance et sa peur. Cette humanité endeuillée qui soudain s'exprime et distingue la captive des dépouilles qui l'entourent, la tragédie l'autorise quand les discours officiels, oraisons funèbres en tête, la nient ${ }^{18}$. Nul doute qu'elle paraisse déplacée ou dérangeante à certains des spectateurs de 415 av. J.-C., car même s'il n'est pas impossible que les Athéniens aient pu s'identifier, après le sac de leur ville par les Perses, aux malheureux Troyens ${ }^{19}$, s'il est évident que le noble caractère d'Andromaque, rendu familier par l'Iliade, lui attire la sympathie et justifie que le public s'émeuve de son chagrin, les guerres médiques sont loin et la situation de l'épouse d'Hector est celle des ennemis d'Athènes en cette fin de siècle : elle est asservie et arrachée à sa cité comme les femmes de Skionè ou de Mélos. Compatir avec ses souffrances implique d'admettre que les victimes des Athéniens ont toutes les raisons de se lamenter.

Un nouvel élément vient peut-être renforcer ce processus complexe d'identification. Je voudrais suggérer que l'entrée en scène du chariot plein d'objets précieux pouvait rappeler au public athénien et à ses

17 Lee, K.H., éd., Euripides. Troades, Basingstoke, 1976, p. 174 ; Halleran M.R., Stagecraft in Euripides, p. 97.

18 C'est la thèse principale de N. Loraux dans La Voix endeuillée (voir notamment p. 87 et suiv. ; voir aussi, à sa suite, $\mathrm{C}$. Dué, The Captive Woman's Lament). La tragédie contrevient ainsi à l'interdiction faite aux familles d'exprimer publiquement leur deuil à la mort d'un soldat, qui est un topos des oraisons funèbres athéniennes. La limitation ou l'interdiction du deuil et du thrène qui l'exprime a pu aussi être inscrite dans la loi : voir Plutarque, Solon, 21, 6 et le début du décret de la «Stèle des Braves » de Thasos, fortement inspiré par le droit athénien (Pouilloux J., Recherches sur l'histoire et les cultes de Thasos, I, Et. Thas.III, 1954, 141 ; Fournier J., Hamon P., « Les orphelins de guerre de Thasos : un nouveau fragment de la stèle des Braves (ca 360-350 av. J.-C.) », BCH 131, 2007, p. 309-381).

19 Sur ce sujet, je suis séduite par les arguments de G. Ferrari (« The Ilioupersis in Athens », HSCP, 100, 2000, p. 119-150). 
hôtes étrangers l'exposition du tribut des alliés, qui avait lieu lors des Grandes Dionysies avant le concours tragique. Nous ne savons que peu de choses sur cette cérémonie, et l'idée que j'avance demeurera une conjecture, mais il vaut la peine de s'y arrêter. Selon Isocrate, le

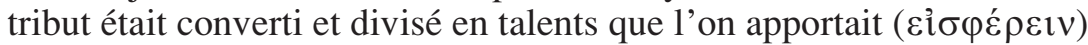
au théâtre pour les y offrir à la vue de tous ${ }^{20}$. Dans un commentaire à ce texte ${ }^{21}$, Raubitschek a développé l'hypothèse que chaque talent était transporté par un homme dans un sac ou un vase. Pour le contenant, il s'appuie sur un bas-relief figurant sur une stèle au-dessus d'un décret relatif au tribut ${ }^{22}$. Quant à la méthode de transport, il l'extrapole à partir de la frise des Panathénées qui montre des jeunes gens portant des vases sur leurs épaules. Le contexte, cette fois, est étranger à la question du tribut, et la conjecture paraît plus faible. Il me semble, pour ma part, qu'on ne peut exclure que le tribut ait été apporté au théâtre sur un ou plusieurs chariots ${ }^{23}$. Si tel était le cas, le poète chercherait alors à associer le butin des vainqueurs grecs au tribut, symbole de la puissance athénienne. En superposant le spectacle des dépouilles troyennes à celui de la contribution des alliés, il pourrait suggérer que la différence entre empire et conquête est mince, qu'il n'y a qu'un pas de la direction légitime de l'alliance à l'asservissement des alliés. La présence de la veuve et de l'orphelin, privés de leur époux et père, privés de leur liberté, peut être une allusion au coût humain de l'empire athénien et aux ravages que les guerres liées à sa survie ou à son extension provoquent dans des familles - grecques, celles-là. Il est clair que si cette hypothèse est juste - je ne peux offrir d'autre preuve que sa vraisemblance et le fait qu'Euripide, comme nous allons le voir, intègre d'autres événements des Grandes Dionysies dans les Troyennes - la signification et la portée de la pièce deviennent plus étroitement, plus précisément politiques que la critique la plus récente n'a voulu le penser, et qu'il faut reconsidérer la référence aux conflits impérialistes menés par Athènes ${ }^{24}$. Il devient dès lors difficile de ne voir dans la tragédie qu'une dénonciation universelle de la guerre, et d'ignorer sa portée ouvertement polémique. Le public est invité à éprouver de la honte, de la souffrance, voire de la peur à la vue de cette scène qui révèle les aspects potentiellement destructeurs de la relation entre Athènes et ses alliés.

\footnotetext{
20 Sur la Paix, 82.

${ }^{21}$ Raubitschek, A.E., « Two Notes on Isokrates », TAPA 72, 1941, p. 356-364.

${ }_{22} \mathrm{IG} \mathrm{I}^{3} 1,68$.

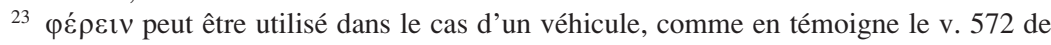
notre pièce.

${ }^{24}$ Voir Croally, N., Euripidean Polemic, p. 232-234 ; Dué, C., The Captive Woman's Lament, p. 147-150.
} 
Jusqu'à présent, je n'ai examiné l'entrée d'Andromaque et d'Astyanax que comme un tableau, un spectacle chargé de signification symbolique. Mais elle possède aussi une forte valeur dramatique, le plus souvent ignorée, en ce qu'elle prépare le coup de théâtre du meurtre de l'enfant. C'est en effet sa relation à son père, condensée dans son nom (le « prince de la ville » est d'abord et avant tout Hector, comme

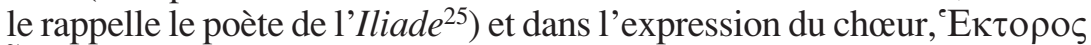
îvis, qui va se révéler fatale. Quant aux armes d'Hector auprès desquelles il se tient, elles joueront un rôle remarquable dans son enterrement, lors duquel objet et corps seront unis dans un destin commun.

Quand Talthybios revient en scène, interrompant la lamentation d'Andromaque et d'Hécube, c'est pour annoncer que les Grecs ont décidé de tuer Astyanax. L'espoir réconfortant que l'enfant pourrait un jour reconstruire Troie est balayé à l'instant même où Hécube l'exprime ${ }^{26}$. L'annonce est destinée à prendre de court le public ${ }^{27}$ aussi bien que les personnages, foudroyés par l'horreur et la gratuité du crime, car aucune allusion à ce meurtre n'a été faite précédemment, ni par les dieux dans le prologue, ni par Cassandre, ni par Talthybios, manifestement surpris. Le retour du héraut au milieu de l'épisode, qui bouleverse le rythme régulier de ses entrées et sorties, souligne le caractère soudain et inattendu de la décision des Grecs. Le meurtre n'a pas été prévu, préparé ni demandé par les dieux, contrairement au sacrifice d'Iphigénie par exemple. Il est d'autant plus tragique qu'il n'est que l'expression d'une décision humaine motivée par la peur ou la haine, qu'aucune nécessité divine ne semble expliquer.

Mon propos n'est pas ici d'insister sur le pathétique du dialogue entre le messager, envahi d'une compassion qui s'exprime par la réticence et la brusquerie, et la malheureuse mère, ravagée par la douleur, déchirée entre la haine des Grecs et la nécessité de contrôler l'expression de sa souffrance pour assurer une sépulture à son fils ${ }^{28}$. Commentateurs et metteurs en scène ont depuis longtemps souligné, expliqué et exploité la puissance émotionnelle de ce moment de théâtre, qui met en jeu des sentiments - douleur, haine, impuissance, pitié - et des rapports de pouvoir universels. C'est peut-être précisément l'universalité de cette scène bouleversante, son immédiateté, qui en ont masqué

$25 \mathrm{Il} . \mathrm{VI}, 403$.

26 Cf. 702-705.

27 On peut discuter sur les attentes du public : le meurtre d'Astyanax, après tout, est déjà présent sous forme de conjecture à la fin de l'Iliade (XXIV, 725-737), et constitue un élément traditionnel de l'Ilioupersis. D'un autre côté, Euripide est coutumier des innovations mythiques. Dans le prologue même des Troyennes, ne fait-il pas de Poséidon un allié des Troyens, en contradiction avec la tradition iliadique ?

${ }_{28}$ Voir le bel article de M. Dyson et K.H. Lee, «The Funeral of Astyanax », p. 17-22. 
la remarquable spécificité. Car ce qui se passe, ce n'est pas seulement que les vainqueurs arrachent son fils à la princesse des vaincus, qu'on sépare par la force une mère de son enfant : les mots de Talthybios et d'Andromaque renvoient avec la plus grande précision à la cérémonie des orphelins de guerre athéniens et à la proclamation du héraut qui a été faite dans le théâtre la veille ou l'avant-veille du concours tragique et l'inversent systématiquement. Nous avons la chance d'avoir conservé, grâce à Lysias et à Eschine, deux allusions complémentaires à la cérémonie et à la proclamation :

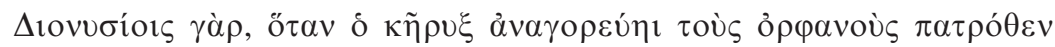

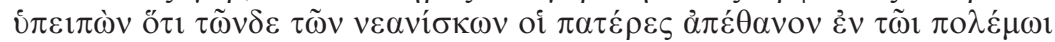

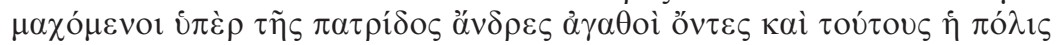

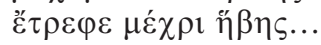

«Lors des Dionysies, lorsque le héraut annoncera, en appelant les orphelins par le nom de leur père : "Voici les jeunes gens dont les pères sont morts à la guerre en combattant en braves pour la patrie, et que la cité a élevés jusqu'à leur majorité, »... » (Lysias, fragment du Contre Théozotidès) ${ }^{29}$

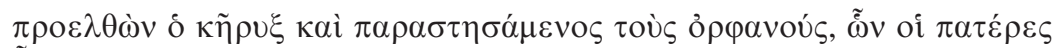

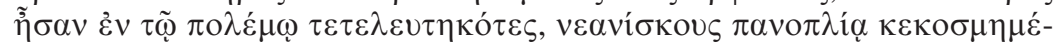

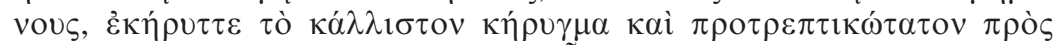

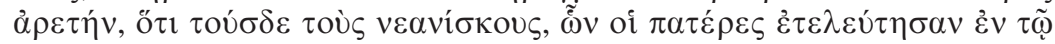

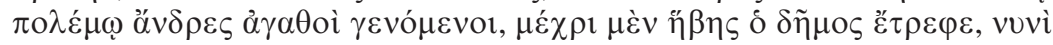

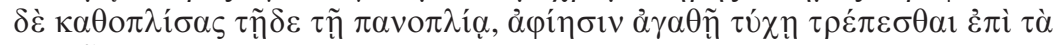

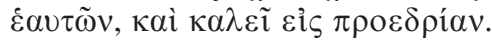

« Le héraut s'avançait, présentait les orphelins dont les pères étaient morts à la guerre, jeunes gens revêtus de l'armure complète, et prononçait la plus belle des proclamations, la mieux faite pour inciter à la vertu : «Le peuple a élevé jusqu'à leur majorité ces jeunes gens dont les pères sont morts en braves à la guerre ; et maintenant, il les arme de cette armure complète, il les envoie chacun mener leur propre vie en les recommandant à la bonne fortune, et les invite à occuper le premier rang au théâtre. »»(Eschine, Contre Ctésiphon, 154) ${ }^{30}$

Le rapprochement de nos deux sources nous autorise à penser que chacun cite, bien que partiellement, le même texte proclamé par le héraut et rappelant les honneurs conférés aux orphelins : outre la

${ }^{29}$ Ed. Bizos M., trad. Gernet L. (CUF, 1955) modifiée par J. Fournier et P. Hamon dans « Les orphelins de guerre de Thasos ».

30 Trad. Martin V. et de Budé G. (CUF, 1952). J'ai modifié légèrement la traduction,

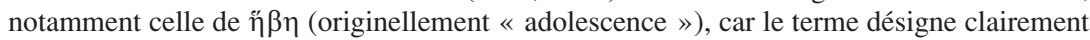
ici le moment, atteint l'année où se déroule la cérémonie et célébré par le rite, où les adolescents, dotés d'une armure, deviennent des hoplites et accèdent au monde et aux responsabilités des hommes adultes. 
trophè qui leur a été allouée jusqu'à ce jour ${ }^{31}$, mentionnée par les deux orateurs, la panoplie et la proédrie ajoutées par Eschine ${ }^{32}$. Le fragment de Lysias précise, pour sa part, que les orphelins sont appelés du nom de leur père et que les Braves ont combattu pour la défense de la patrie.

Cela signifie que les orphelins parvenus à l'âge d'homme ( $\eta \beta \eta)$ qui ont reçu cette année-là leur panoplie sont assis aux premiers rangs du théâtre quand les tragédies, et les Troyennes en particulier, sont représentées. Derrière eux, les pères qui vont au combat pour défendre la cité en sachant que, s'ils venaient à mourir, l'Etat prendrait soin de leurs fils ${ }^{33}$. Tous ont sous les yeux l'orphelin du plus grand des guerriers troyens, qui leur a été désigné comme le « rejeton d'Hector ». De la bouche du héraut, ils apprennent que cet enfant sera tué parce que

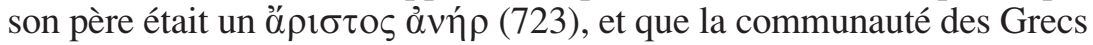
- Talthybios insiste sur le caractère collectif de la décision (711) et de

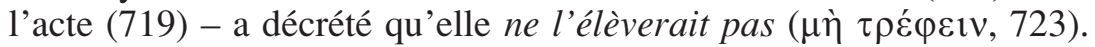
Il ne sera pas «envoyé » ( $\dot{\alpha} \varphi-i ́ n \sigma ı)$ mener sa propre vie mais

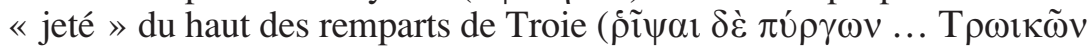
ö́ro, 725). Talthybios, comme le héraut athénien, s'efforce même de contrôler la réaction du public (ici, Andromaque) en lui interdisant de

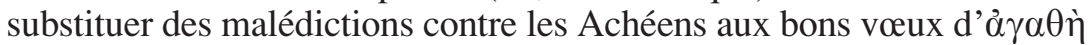
$\tau u ́ \chi \eta$ que les orphelins athéniens reçoivent de leur cité :

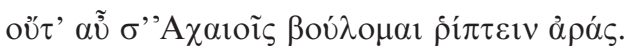

« Je t'invite à ne pas lancer d'imprécations contre les Achéens » (734)

Ce vers appartient au discours suivant l'annonce du décret, dans lequel Talthybios conseille à Andromaque de ne pas s'y opposer. Usant de l'argument de la force que Thucydide développe dans le dialogue des Athéniens et des Méliens ${ }^{34}$, le héraut tente d'empêcher la princesse troyenne, mue par le chagrin et la colère, d'en venir à des extrémités

31 Voir Stroud R.S., « Greek Inscriptions. Theozotides and the Athenian Orphans », Hesperia 40, 3, 1971, p. 280-301.

${ }_{32}$ Vraisemblablement sur le modèle athénien, des privilèges similaires sont accordés aux orphelins de guerre de Thasos au milieu du IV siècle : proédrie aux concours (1. 14), panoplie (le décret détaille sa composition 1. 18-19), complétées d'une invitation au banquet que la cité donne en l'honneur des Braves (1.9-10). La trophè, elle, est limitée à ceux des orphelins qui sont nécessiteux (1. 26). Une proclamation officielle de ces honneurs est prévue lors des Héracleia (1. 20). Voir Fournier J. et Hamon P., « Les orphelins de guerre de Thasos".

33 Voir Ober, J., Democracy and Knowledge. Innovation and Learning in Classical Athens, Princeton, 2008, p. 256-258.

34 Thuc. HGP, IV, 85-113. Il est intéressant de noter qu'Andromaque, sur le point de maudire les Grecs pour leurs actes barbares, se reprend et dévie sa haine vers Hélène, sur qui elle appelle la mort. 
et cherche à préserver un semblant de normalité : l'enfant mort sera enterré et pleuré, à condition qu'Andromaque cède à ses menaces et se montre, comme toujours, une femme digne, une femme capable de comprendre « quand il faut vaincre et quand il faut céder la victoire » ${ }^{35}$. Le consensus qui se forme autour des orphelins athéniens quand la cité assemblée les honore est donc remplacé par une acceptation forcée qu'Andromaque exprime en de déchirants impératifs au moment où elle se résout à abandonner son enfant :

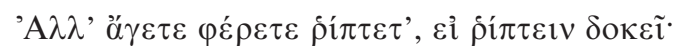

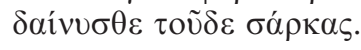

« Eh bien ! emmenez cet enfant, emportez-le, précipitez-le, s’il vous plaît de le précipiter ! Faites un festin de sa chair ! » (774-775)

La cérémonie des orphelins athéniens est donc intégrée à la pièce et rigoureusement inversée. Contrairement aux jeunes gens du public, Astyanax n'atteindra jamais l'âge d'homme et n'aura jamais la possibilité d'égaler son père. Il ne participera pas aux banquets pour y boire avec les adultes, mais est assimilé par sa mère à un animal sacrifié, partagé et mangé lors d'un festin (dais) monstrueux ${ }^{37}$. Dans la lamentation qui suit la proclamation de Talthybios, Andromaque attribue la mort

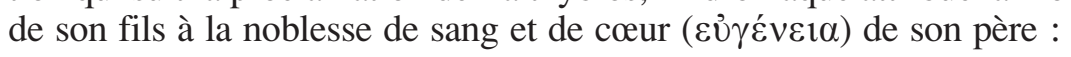

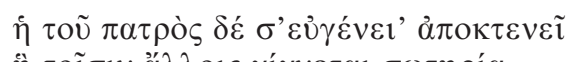

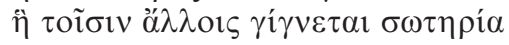

«C'est la noblesse de ton père qui va te tuer, cette noblesse qui aux autres apporte le salut. » $(742-743)^{38}$

L. Parmentier et D. Kovacs, entre autres, considèrent que ces vers ne renvoient qu'à Hector, et ő' $\lambda \lambda$ or $\varsigma$ aux Troyens qu'il protégeait tant qu'il était en vie, mais ceci les conduit à transformer le présent $\gamma^{\prime} \gamma v \varepsilon-$ $\tau \alpha \imath$ en passé. K.H. Lee développe des arguments convaincants pour

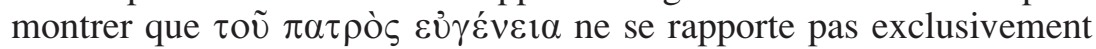
à la valeur d'Hector, mais à la noblesse paternelle en général, ce qui permet d'expliquer le présent de $\gamma^{\prime} \gamma v \varepsilon \tau \alpha 1^{39}$. Si l'on adopte cette 656.

35 C'est ainsi qu'Andromaque définit elle-même l'art d'être une bonne épouse v. 655-

${ }^{36}$ Trad. Parmentier modifiée.

${ }^{37}$ L'image des Grecs partageant et mangeant la chair d'Astyanax rappelle les peurs et les menaces de cannibalisme exprimées dans l'Iliade (le passage le plus pertinent ici est sans doute, pour la référence au père d'Astyanax, XXII, 346-348 où Achille regrette de ne pouvoir dévorer son adversaire troyen) ; mais elle évoque aussi la lamentation d'Andromaque à la mort d'Hector (XXII, 494-498), dans laquelle elle imagine son fils orphelin chassé des banquets par des adultes qui ne sont pas son père.

38 Trad. Parmentier modifiée.

39 Voir Lee K.H., Troades, ad v. 742-743. 
perspective, qui sont les « autres » à qui la noblesse paternelle apporte le salut ? Les enfants des Achéens, sans doute, et en premier lieu ceux d'Ulysse, instigateur de la décision grecque, qu'Andromaque maudit en souhaitant que les arguments de leur père l'emportent aussi pour eux et leur coûtent la vie comme ils la coûtent à Astyanax $(724)^{40}$. Mais j'irais jusqu'à suggérer qu'Euripide peut ici faire référence directement à son

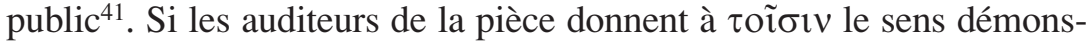
tratif que le mot a chez Homère et peut encore avoir dans la langue de

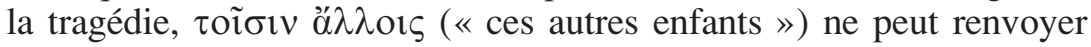
qu'aux fils présents parmi les spectateurs, car il n'y a pas d'autre garçon ou jeune homme sur la scène auquel la locution puisse faire allusion. Andromaque comparerait donc directement le destin de son fils à celui des fils des Athéniens, et en particulier des orphelins assis aux premiers rangs. La valeur paternelle, ce topos des discours officiels si propre à enflammer le courage non seulement des fils, mais de tous les citoyens, a été pervertie dans la tragédie ; elle est devenue la justification inattendue d'un meurtre, et l'instrument de l'assassinat d'Astyanax.

Euripide ne se contente pas d'exploiter ces allusions et inversions verbales. Il en fait, lors du dernier épisode, un spectacle qui donne chair et corps à la proclamation du héraut et à la décision abstraite des Grecs $^{42}$. Lorsque Talthybios rapporte à Hécube le cadavre du garçon, qui a été jeté du haut des remparts, pour qu'elle le pleure et prépare son enterrement, le public voit entrer sur scène le corps de l'enfant allongé sur un bouclier et porté par des soldats grecs ${ }^{43}$. C'est Andromaque qui a demandé qu'Astyanax reçoive pour linceul le bouclier de son père, ne pouvant supporter l'idée que l'arme, exhibée dans la demeure de son nouveau maître, lui rappelle éternellement Hector et son bonheur conjugal disparu ${ }^{44}$.

40 Le parallèle ainsi établi entre la valeur d'Hector et celle d'Ulysse est bien sûr amèrement ironique, car l'eugeneia du fils de Laerte est démentie par toutes ses actions, dans le Palamède, où il calomnie un innocent, comme dans les Troyennes, où il obtient qu'on tue un enfant.

41 Taplin, O., «Fifth Century Tragedy and Comedy : A Synkrisis »JHS 106, 1986, p. 166-167, considère que les références au public, qui perturbent l'illusion théâtrale, sont exclusivement du registre de la comédie. Mais Pollux, à propos de la parabase (Onomasticon, IV, 111), a une allusion intrigante au fait qu'Euripide, comme les poètes comiques, fait souvent parler le choeur en son nom. Il n'est donc pas exclu qu'il rompe lui aussi, à l'occasion, l'illusion du théâtre.

42 L'une des trouvailles du film de M. Cacoyannis (The Trojan Women, 1974) est d'accomplir la même matérialisation du crime en montrant, par un trucage et une caméra subjective, le moment de la chute.

43 Halleran (Stagecraft in Euripides, p. 100) remarque que le discours du chœur décrivant l'entrée, contrairement à l'usage, ne mentionne pas le personnage qui entre, Talthybios. Cela a pour effet de concentrer l'attention exclusivement sur le cadavre.

44 v. $1133-1142$. 
Il se peut que le modèle premier de ce tableau soit celui des guerriers morts que l'on emportait du champ de bataille sur leur bouclier. Il est vrai que cet usage n'est pas attesté pour l'armée athénienne, et que nous n'avons pas de trace de soldat enterré avec ses armes en Attique après le début du $\mathrm{VII}^{\mathrm{e}}$ siècle, mais Plutarque mentionne plusieurs fois que la coutume existait chez les Spartiates ${ }^{45}$, et il se peut que cette image soit présente à l'esprit des spectateurs. Le spectacle du petit corps immobile, le contraste qu'il forme avec l'impressionnant bouclier paternel et les corps, animés ceux-là, des hommes qui le portent, évoquerait alors non seulement la brutalité du traitement que les adultes ont infligé à l'enfant innocent ${ }^{46}$, mais aussi, à travers l'image du guerrier mort qu'Astyanax ne sera jamais, l'anéantissement de son avenir comme héros et nouveau défenseur de Troie.

L'association d'Astyanax et du bouclier parachève par ailleurs la perversion de la cérémonie des orphelins athéniens, au cours de laquelle ils reçoivent une panoplie. En tant qu'élément des hopla d'Hector, le bouclier fonctionne comme une métonymie de panoplie, même si ce n'est pas le mot ö $\pi \lambda$ ov qui le désigne dans le texte, mais les plus poé-

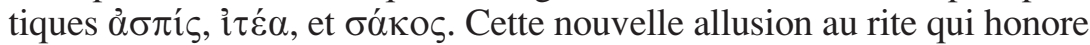
les jeunes Athéniens et les encourage à suivre les traces de leur père rappelle cruellement au public que le fils d'Hector ne vivra pas pour accomplir ce que ses parents et sa cité attendaient de lui, qu'on l'a empêché d'imiter son père ${ }^{47}$. Le plus ironique est sans doute qu'Hector, bien que brave et dévoué à sa cité, a été vaincu, et que son exemple ne devrait pas inquiéter les Grecs ${ }^{48}$.

Le geste d'Andromaque s'assurant que le fils obtienne l'arme du père constitue un autre écart important par rapport à l'octroi de la panoplie aux jeunes Athéniens. C'est un geste de séparation destiné à permettre l'oubli vital : pour pouvoir continuer à vivre et supporter les contraintes, les exigences de sa propre destinée comme concubine de Néoptolème, Andromaque a résolu de laisser derrière elle les fantômes de son passé anéanti ${ }^{49}$. Or la parade des orphelins en armes a précisé-

\footnotetext{
45 Prop. Lac. 235, A, 1 suiv. ; 241, F, 4-6.

46 Voir v. 765 et 1165 . Le caractère innocent et inoffensif d'Astyanax s'oppose vivement à la culpabilité et à la rouerie d'Hélène, qui font l'objet de la scène d'agôn pendant laquelle l'enfant est assassiné hors scène.

47 Inversement, dans l'Ajax de Sophocle, le héros lègue lui-même son bouclier à son fils (v. 574-577) au terme d'un discours par lequel il l'incite à imiter son père et l'assure que des proches l'accompagneront jusqu'à l'âge adulte. Je traiterai ailleurs des questions d'intertextualité dans Les Troyennes.

${ }^{48}$ C'est du moins le constat amer que fait Hécube, indignée de la peur et de la lâcheté des Achéens (v. 1158-1166).

${ }^{49}$ L'abandon du bouclier d'Hector représente le résolution du dilemme d'Andromaque : « Si je rejette de ma pensée le visage aimé d'Hector disparu pour ouvrir mon cœur à l'époux d'aujourd'hui, je paraîtrai lâche au disparu ; si c'est l'autre que je repousse, je m'attirerai la haine de l'homme qui est mon maître » (v. 661-664).
} 
ment pour but de rappeler la gloire des pères à leurs fils comme à l'ensemble des citoyens, d'en perpétuer la mémoire, de la reproduire dans la génération suivante, et de célébrer ainsi la permanence de la communauté. Mais si Andromaque réunit Hector et Astyanax, c'est dans le monde des morts qui l'exclut, elle, et aux pieds d'une Troie vide de citoyens dont la disparition est imminente. Elle consacre en quelque sorte l'éclatement de sa famille et de sa cité.

Interpréter l'usage que fait Euripide du rituel des orphelins athéniens et la manière dont il caractérise ainsi le meurtre d'Astyanax, comprendre quelles réactions cela pouvait faire naître dans le public de 415 av. J.-C. ne sont pas choses aisées. Le dévoiement de la cérémonie et de la proclamation, sans doute, contribue à faire de l'action des Grecs quelque chose d'anormal. L'anomalie ne réside pas seulement dans le problème éthique que constitue le meurtre d'un enfant innocent; de façon beaucoup plus spécifique, elle est caractérisée par la perversion d'un rituel civique que tous les membres du public identifient sans encombre, puisqu'ils y ont assisté la veille. Cela ne signifie pas pour autant que tout auditeur devait juger la décision des Grecs immorale ou infondée, car après tout, Astyanax n'est pas un orphelin grec, mais un ennemi et un barbare. Il me semble néanmoins que cette objection ${ }^{50}$ est contrée dans le texte-même par la fameuse exclamation d'Andromaque dénonçant les Grecs aux crimes barbares ( ${ }^{\circ} \Omega \beta \alpha ́ \rho \beta \alpha \rho$ '

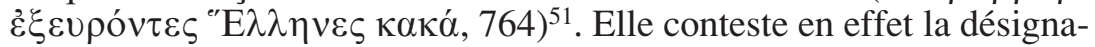
tion des Grecs et des barbares (les gens qui pervertissent des rites grecs doivent être non-grecs) et donne à cette distinction un contenu moins ethnique que moral, que renforce l'insistance sur la responsabilité des auteurs du crime par opposition à l'innocence de la victime ( $\tau$ í $\tau o ́ v \delta \varepsilon$ $\pi \alpha \tilde{i} \delta \alpha \kappa \tau \varepsilon i ́ v \varepsilon \tau$ o o $\delta \dot{\varepsilon} v$ aítıov ; 765). La réaction de Talthybios, qui mêle l'embarras et la brusquerie à la pitié, guide également la réaction du public externe. Le meurtre peut donc apparaître comme d'autant plus choquant, scandaleux et pathétique que les Grecs refusent à Astyanax exactement ce qu'ils (ou plutôt les Athéniens) accordent à leurs propres orphelins.

Quelle est, maintenant, la portée critique de cette utilisation de la loi et du rite athéniens ? Dans quelle mesure sert-elle la contestation de l'idéologie guerrière sur laquelle ils reposent ? N. Croally a examiné avec soin la manière dont « Euripide exploite le contexte de la guerre et de la crise qu'elle entraîne pour révéler les problèmes liés aux

\footnotetext{
50 Objection qui m'a été faite plusieurs fois quand j'ai présenté oralement ce travail.

51 Voir Saïd S., « Grecs et barbares dans les tragédies d'Euripide. La fin des différences ? », Ktèma, 9, 1984, p. 27-53.
} 
polarités constitutives de l'idéologie athénienne $»^{52}-$ il entend par là les distinctions entre Grecs et barbares, amis et ennemis, hommes libres et esclaves, hommes et femmes, etc. - « en créant dans le temps et l'espace un monde dramatique [spécifique] et en faisant un usage croissant de l'auto-référence $»^{53}$. La découverte que cette autoréférence inclut l'intégration de la cérémonie des orphelins au monde tragique des Troyennes nous permet de renforcer la thèse de Croally, qui s'appuie essentiellement sur les aspects intellectuels de la pièce, et de l'ancrer dans le drame proprement dit. En combinant le rite civique qui les a rassemblés au théâtre il y a peu à la tragédie d'Astyanax, mise en scène dans le même lieu, Euripide oblige les spectateurs à s'interroger sur le lien entre les deux événements, à comparer à la fois les situations, leur signification, et leurs propres réactions. La réflexivité que cela impose peut se révéler particulièrement dérangeante.

Au cours de leur propre cérémonie, les Athéniens ont célébré, à travers leurs orphelins, la continuité d'une communauté civique qui transcende les deuils individuels et privés. Ils ont fait la preuve de leur capacité à restaurer les liens familiaux affectés par la guerre en substituant aux pères disparus le soutien et la protection de la cité ; ils sont parvenus à perpétuer la valeur hérö̈que des morts en faisant de leurs fils des citoyens-soldats. La mort d'Astyanax, quant à elle, est encadrée par deux tirades où Hécube associe la continuité de Troie à l'existence de l'enfant. Avant que Talthybios n'entre en scène pour proclamer le décret des Grecs, la vieille reine tente de réconforter Andromaque, de lui donner une raison de préférer la survie, fût-ce aux mains de Néoptolème, à la mort qui tout efface :

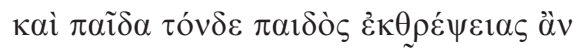

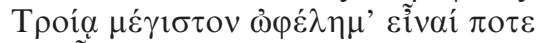

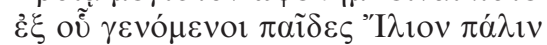

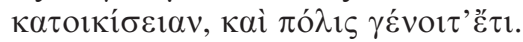

« Tu pourrais ainsi élever jusqu'à l'âge d'homme ce fils de mon fils, afin qu'il rende un jour à Troie les plus grands services, lui dont les fils, plaise aux dieux, rebâtiront Ilion - puisse la cité renaître grâce à eux ! »(702-705) ${ }^{54}$

52 «Euripides exploits the context of war, and of the consequent crisis, to show the problems of polarities constitutive of Athenian ideology », in Euripidean Polemic, p. 252253.

53 «...the spatial and temporal constitution of [a distinctive] dramatic world and the increasing use of self-reference », Euripidean Polemic, p. 253.

${ }^{54}$ Le texte est difficile. Je suis l'édition de Parmentier (Euripide. Les Troyennes, Iphigénie en Tauride, Electre, Paris, 1925), qui préfère à juste titre la leçon $\dot{\varepsilon} \xi$ oữ à $\dot{\varepsilon} \kappa \sigma o \tilde{\text {, à }}$

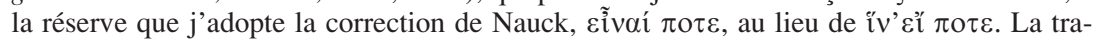
duction est mienne. 
Achever l'éducation d'Astyanax ( $\dot{\varepsilon} \kappa-\tau \rho \dot{\varepsilon} \varphi \varepsilon \imath v)$, l'amener à la majorité, en faire un homme, apparaît aux yeux d'Hécube comme une nécessité collective, car dans le monde aristocratique du mythe, c'est la continuité de la lignée royale - le fils du fils $(\pi \alpha \tilde{i} \delta \alpha \ldots \pi \alpha \imath \delta$ ò $\zeta)$ engendrera à son tour des fils $(\grave{\varepsilon} \xi$ oũ $\gamma \varepsilon v o ́ \mu \varepsilon v o r ~ \pi \alpha \tilde{i} \delta \varepsilon \varsigma)$ - qui assure la survie et la permanence de la communauté. Hécube veut croire, ou du moins tente de persuader Andromaque qu'en tant que mère aimante et bonne épouse pour son nouveau maître, elle aura le pouvoir d'assurer à l'enfant la sécurité d'un nouveau foyer, de lui offrir une vie pleine et par conséquent d'assurer un avenir à la cité. Mais le message de Talthybios vient brutalement la contredire : le choix ne lui appartient pas, elle ne peut que se soumettre aux maîtres grecs qui, eux, refusent d'élever l'enfant et veulent annihiler Troie. Hécube revient sur ce sujet quand elle pleure son petit-fils, mais cette fois pour souligner la rupture que constitue sa mort. Les vœux se sont mués en regrets :

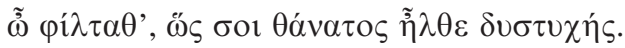

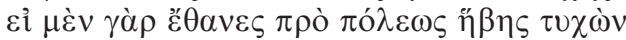

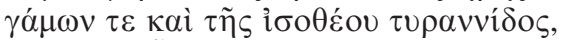

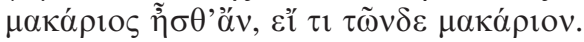

«Enfant chéri, combien la mort pour toi a frappé sans pitié ! Si tu étais tombé pour la patrie, après être parvenu à l'âge d'homme, à l'hymen et à la royauté qui nous égale aux dieux, tu aurais été heureux, s'il y a là quelque bonheur. » $(1167-1170)^{55}$

Astyanax n'a pas atteint l'hèbè ; il ne connaîtra ni le mariage ni la royauté, ce qui signifie que famille et Etat disparaissent avec lui. Dans son désespoir, Hécube en vient à regretter que, contrairement à son père ou aux orphelins athéniens, il n'ait pas eu la possibilité de tomber pour la patrie. Cette mort, du moins, lui aurait valu d'obtenir une gloire similaire à celle de son père, et lui aurait laissé le temps de jouir d'une vie d'homme. Elle aurait été préférable à la fin horrible qui est la sienne. Ce passage a été interprété comme un revirement par rapport au scepticisme qu'Hécube a opposé dans le premier épisode à Cassandre lorsque celle-ci cherchait à la convaincre qu'Hector et Troie, s'étant battus et sacrifiés pour une cause plus noble, étaient en fin de compte plus heureux ( $\mu \alpha \kappa \alpha \rho$ เó $\varepsilon \rho \rho)$ que les Achéens ${ }^{56}$. Son changement de

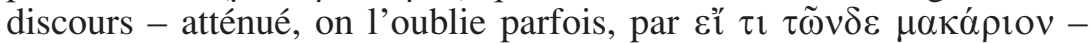
peut s'expliquer, me semble-t-il, si l'on prend en compte la comparaison implicite qu'elle trace entre la mort de l'enfant et celle de son père. Confrontée à un paroxysme de violence qui bouleverse les normes, elle est désormais prête à admettre que, au regard du meurtre d'Astyanax,

55 Trad. Parmentier modifiée.

56 Voir 466-510. 
la mort d'Hector au combat, du moins, entre dans le cadre de la vie normale d'un homme, qu'elle lui a laissé le temps d'accomplir des activités vitales et de connaître quelque joie, et qu'elle lui a valu une réputation d'excellent défenseur de sa cité. Lui, du moins, a laissé un enfant derrière lui.

Mais cette consolation s'évanouit bien vite. Hécube poursuit sa lamentation en détaillant les blessures sanglantes que la chute du haut des remparts paternels $(\tau \varepsilon i ́ \chi \eta ~ \pi \alpha \tau \rho \tilde{\omega} \alpha, 1174)$ a causées à la tête de l'enfant, à ses mains, autrefois si semblables à celles de son père et désormais brisées (1178-1179), à sa bouche, naguère si confiante en l'avenir, si pleine de promesses (1180-1184). Les Grecs ont jeté le garçon : ce n'est plus un mot abstrait, mais une réalité tangible dont les paroles d'Hécube invitent les spectateurs à visualiser les effets. Comme lorsqu'elle opposait à l'éloge paradoxal et désincarné que Cassandre faisait de Troie la matérialité physique de sa propre expérience, de sa propre souffrance ${ }^{57}$, la vieille reine balaie le rêve fugitif d'un Astyanax adulte et heureux en ramenant sa lamentation et l'attention du public aux souffrances du corps ${ }^{58}$. Les paroles qu'elle adresse au petit cadavre sont extrêmement pathétiques, et l'on éprouve d'abord de la pitié devant la vulnérabilité de l'enfant.

Mais sous les mots tendres, Hécube révèle aussi la force subversive du spectacle. La tête fracassée ${ }^{59}$ est la preuve de l'échec d'Hector, incapable de protéger son fils, et de la vanité de sa lutte pour défendre les remparts de la cité, aujourd'hui fatals à son prince et seul héritier ; elle indique qu'Apollon a abandonné Troie, lui qui en avait bâti les murs; elle rappelle enfin le temps disparu où Andromaque prodiguait à son fils des soins aimants : la mère n'a pas même eu la liberté de rester à Troie pour pleurer son enfant. Les doigts et les poignets désarticulés incarnent quant à eux à la fois la ressemblance du père et du fils et la rupture du lien entre eux, l'absence de transmission de l'un à l'autre. La bouche désormais muette, les promesses non tenues témoignent de la perturbation qu'ont subie, sous le coup de la violence, à la fois le cours normal du temps, dans lequel il revient au petit-fils d'enterrer sa grand-mère et non l'inverse, et l'usage normal du rite, selon lequel le garçon devrait couper ses boucles de cheveux pour honorer les morts et non avoir la tête rasée par le tranchant des remparts ${ }^{60}$,

\footnotetext{
${ }^{57}$ Ibid.

${ }^{58}$ Froma Zeitlin voit là un trait caractéristique des personnages féminins de la tragédie : Zeitlin, F., « Playing the Other: Theater, Theatricality, and the Feminine in Greek Drama », in Winkler, J., Zeitlin, F., Nothing to Do with Dionysos? Athenian Drama in its Social Context, Princeton, 1990, p. $1 ; 71$ et suiv.

${ }^{59}$ Sur l'importance de la tête dans l'imagerie et le symbolisme troyens dans l'Iliade, voir Brillet-Dubois, P., « Les dons divins faits aux Troyens », Gaïa 4, 1999, p. 9-16.

${ }^{60}$ Comparer $1173-1175$ et $1182-1184$.
} 
selon lequel il devrait remporter une couronne pour « une victoire sur [ses] compagnons dans les courses hippiques ou au tir à l'arc » (12091210) et non être couronné pour ses funérailles.

Avec la vie d'Astyanax, c'est non seulement la communauté troyenne, mais le sens-même de la lutte de ses hommes que les Grecs ont anéantis : le risque de la destruction totale était au cœur de la guerre qu'ils ont menée avec tant de fougue et d'orgueil, si bien qu'Hécube juge bon de couronner le bouclier d'Hector, lui aussi, non pour les victoires et les trophées qu'il a permis au héros de remporter, mais parce qu'il meurt paradoxalement avec l'enfant (1221-1223). Les guerriers troyens, en allant au-devant de la mort, ont laissé leurs orphelins sans personne pour les défendre et ont exposé la cité à l'anéantissement.

A travers le cas-limite de la défaite troyenne et d'Astyanax - en tant qu'enfant royal unique, il concentre dans le mythe les attentes que la cité athénienne fait reposer sur ses générations de jeunes gens -, les Athéniens du public sont invités à entrevoir la menace qui se cache au cœur de leur propre idéologie et à s'interroger sur la pérennité de leur cité, qui repose sur l'idée implicite qu'elle ne peut être défaite. Que se passerait-il si les Athéniens étaient vaincus ? Que deviendrait Athènes si des ennemis victorieux lui infligeaient le traitement réservé aux Troyens - ou aux Méliens ? Certains spectateurs pourraient même pousser plus loin : et si « envoyer les jeunes gens poursuivre leur carrière » à la guerre après les avoir honorés et armés était potentiellement équivalent à « jeter les jeunes gens du haut des remparts »? Le verbe f́í $\tau \omega$, qu'Euripide substitue avec tant d'insistance à ĩ $\mu$, ne réalise-t-il pas la signification la plus violente de í faire des soldats et célébrer le sacrifice ultime comme le plus glorieux des services rendus à la patrie se révélait en fin de compte source d'auto-destruction?

Ces interrogations, Euripide les fait naître par les tours et ambiguïtés du langage, par la richesse symbolique du tableau d'Astyanax couché dans le bouclier de son père, mais aussi en exploitant la forme, les thèmes et la puissance expressive de la lamentation rituelle. Les victoires et le mariage qui n'auront jamais lieu sont des topoi du genre lorsque le chant funèbre s'adresse à des enfants ou des jeunes gens morts avant d'avoir atteint l'âge adulte. Le point de vue féminin des pleureuses est plus complet que celui de l'idéologie masculine qui s'exprime dans la cérémonie des orphelins et privilégie le rôle guerrier des hommes. La lamentation, elle, évoque aussi les autres aspects de la vie : le mariage, la famille, les activités pacifiques, que les proclamations et oraisons officielles oblitèrent. Après la célébration de l'identité civique au début des Grandes Dionysies, les femmes de Troie en deuil viennent rappeler au public athénien qu'un homme joue plus 
d'un rôle dans le monde et que lorsqu'il s'expose au combat, ce n'est pas seulement une vie de soldat qui est en jeu.

Certains commentateurs ${ }^{61}$ voient dans l'exécution du rite funéraire lors du dernier épisode des Troyennes un moment, bref mais bienvenu, d'apaisement et de normalité. Ce mort-là, du moins, est traité comme il se doit ${ }^{62}$, et la lamentation féminine, que rien ne vient interrompre, restaure un semblant d'ordre au milieu des tourments que subissent les captives. La pièce peut dès lors être interprétée comme n'étant pas absolument nihiliste. Il est vrai que grâce à la compassion de Talthybios, Astyanax reçoit des funérailles décentes des mains de sa grandmère. Mais j'espère avoir montré que cet acte d'amour réparateur n'est pas destiné à atténuer l'horreur du crime ni à apaiser les interrogations soulevées par l'ensemble de la pièce. Les funérailles de l'enfant ne sont pas seulement prématurées, elles remplacent ou pervertissent d'autres rites qui auraient dû se dérouler : la célébration de l'accession de l'orphelin à l'âge d'homme, son couronnement comme vainqueur, son mariage. Le problème ne tient pas à l'exécution du rite funéraire, mais plutôt au fait qu'il empêche l'exécution de ces autres cérémonies, fondamentales pour l'existence et la pérennité de la cité. L'inversion très spécifique de la cérémonie des orphelins de guerre se distingue des topoi sur la vie brisée des enfants morts trop jeunes, et constitue, je crois, l'élément d'auto-référence qui donne à la scène son caractère particulièrement choquant. C'est par cette perversion d'un événement civique familier et récent que les Athéniens sont amenés à faire le lien entre le destin d'Astyanax et leur propre monde, leur propre futur, à s'identifier non seulement avec les Grecs victorieux, mais aussi avec Troie, la cité condamnée dont les idéaux de dévouement et de gloire étaient si proches des leurs, à réfléchir enfin à leur propre idéologie, à ce qu'elle implique, et en particulier à la façon dont elle laisse dans l'ombre ou sublime jusqu'à l'intenable paradoxe les dommages causés par la guerre à Athènes comme à ses ennemis. A travers Astyanax, c'est l'ensemble des valeurs attachées à la guerre qui se trouve mis en cause : la valeur et le sens de la victoire, de la gloire et du dévouement à la patrie. La menace que constitue l'idéologie guerrière pour la continuité et la cohésion de la cité, et en fin de compte, pour son existence, est dévoilée au grand jour. Est-il surprenant que cette leçon pénible n'ait pas permis à Euripide de remporter le concours ?

Pascale Brillet-Dubois

Université de Lyon - Université Lumière-Lyon 2 HiSoMA (UMR 5189) - Maison de l'Orient et de la Méditerranée

\footnotetext{
${ }^{61}$ Voir par ex. Dyson, M., Lee, K.H., « The Funeral of Astyanax », p. 28.

${ }^{62}$ Les Troyennes font ailleurs allusion aux corps des hommes gisant sans sépulture (599-600).
} 
